\title{
ALMOST COMMUTING MATRICES NEED NOT BE NEARLY COMMUTING
}

\author{
MAN-DUEN CHOI
}

(Communicated by John B. Conway)

Dedicated to Chandler Davis on the occasion of his sixtieth birthday

\begin{abstract}
Let $\mathcal{M}_{n}$ be the collection of $n \times n$ complex matrices with the Hilbert-space-operator norm. There exist two concrete matrices $A, B \in \mathcal{M}_{n}$ with $\|A\|=1-1 / n,\|B\| \leq 1,\|A B-B A\| \leq 2 / n$, but $\|A-R\|+\|B-S\| \geq$ $1-1 / n$ for all commuting pairs $R, S \in \mathcal{M}_{n}$. It is shown explicitly that there is a natural obstruction which prevents almost commuting matrices to get close to any commuting pairs.
\end{abstract}

1. Introduction. This paper provides a negative solution to an old problem in linear algebra and operator theory.

MAIN PROBLEM. (Must almost commuting matrices be nearly commuting?) For each $\varepsilon>0$, is there a $\delta=\delta(\varepsilon)>0$ such that for each positive integer $n$, if $A, B \in \mathcal{M}_{n}$ with $\|A\| \leq 1,\|B\| \leq 1$ and $\|A B-B A\|<\delta$, then there exist $R, S \in \mathcal{M}_{n}$ with $R S=S R$ and $\|A-R\|+\|B-S\|<\varepsilon$ ?

Herein, the constant $\delta=\delta(\varepsilon)$ should be independent of the integer $n$; otherwise, affirmative answers with $\delta=\delta(\varepsilon, n)$ have appeared in $[\mathbf{1}, \mathbf{1 0}, \mathbf{1 1}]$.

Owing to the expository communications by Halmos $[8, \S 7]$ and others (e.g., $[1,10,11,12])$, the problem above has aroused much interest in recent research. Several cognate problems (as analogues of the main problem) have already been settled in the negative direction. In particular, Berg and Olsen [4] presented an index obstruction for the infinite-dimensional analogue (see also [2]), while Voiculescu [13] suggested a possible second-cohomological obstruction for the unitary-matrix analogue (see also Loring's $K$-theoretical explanation [9]). Most notably, Davidson's intensive study [7] on the analogue for Hermitian matrices has given rise to a quantitative version of the Brown-Douglas-Fillmore extensions (see [3]). Thus, the conquest of the Main Problem is close at hand, but special techniques in $[\mathbf{4}, \mathbf{7}$, 9, 13] may be too special for general matrices with no special structure. In this paper, we propose to investigate a simple invariant of general matrices. Then, with facility in $2 \times 2$ matrix-operator manipulation, we are ready to explain why almost commuting matrices can be "extremely far" away from all commuting pairs.

Received by the editors July 1, 1986 and, in revised form, November 17, 1986.

1980 Mathematics Subject Classification (1985 Revision). Primary 47A55, 15A27; Secondary 47 B47.

Key words and phrases. Almost commuting matrices, Hilbert-space-operator norm, signature of a matrix, commuting approximant, pertubation.

This research was supported in part by NSERC of Canada.

(C) 1988 American Mathematical Society $0002-9939 / 88 \$ 1.00+\$ .25$ per page 
2. Preliminaries. In this paper, the letter $I$ stands for the identity matrix with ones along the diagonal and zeros elsewhere (the order of $I$ should be clear from the context). Let $\mathcal{M}_{n}$ denote the collection of all $n \times n$ complex matrices with complex entries. If $T=\left[t_{i j}\right] \in \mathcal{M}_{n}$, then the adjoint $T^{*}=\left[\bar{t}_{j i}\right]$, the trace of $T$ is $\sum_{j=1}^{n} t_{j j}$, and the characteristic polynomial for $T$ is $\operatorname{det}(T-\lambda I)$. The notation $\|T\|$ refers to the Hilbert-space-operator norm; thus if $T=\left[t_{i j}\right] \in \mathcal{M}_{n}$, then

$$
\begin{aligned}
\|T\| & =\sup \left\{\|T \nu\|:\|\nu\| \leq 1, \nu \in \mathbf{C}^{n}\right\} \\
& =\sup \left\{\left(\sum_{i}\left|\sum_{j} t_{i j} \nu_{j}\right|^{2}\right)^{1 / 2}: \sum\left|\nu_{j}\right|^{2} \leq 1\right\} .
\end{aligned}
$$

For each $T \in \mathcal{M}_{n}$, the spectrum of $T$, denoted by $\sigma(T)$, is the collection of all eigenvalues of $T$. We write $\sigma_{+}(T)$ (respectively $\sigma_{-}(T)$ ) for the collection of eigenvalues which belong to the open right plane $\{z \in \mathbf{C}: \operatorname{Re} z>0\}$ (resp. the open left half-plane $\{z \in \mathbf{C}: \operatorname{Re} z<0\})$. The signature of $T$ is

$$
\operatorname{sgnt} T=\#\left(\sigma_{+}(T)\right)-\#\left(\sigma_{-}(T)\right)
$$

where \# is the number of elements with multiplicity counted. In particular, if $J$ is a selfadjoint unitary (i.e., $J=J^{*}=J^{-1}$ ), then $J$ can have only \pm 1 as eigenvalues, and thus sgnt $J=\operatorname{trace} J$.

Next, we present several simple lemmas.

LEMMA 1. Let $n$ be any fixed positive integer. Then the signature is a continuous function from $\left\{T \in \mathcal{M}_{n}: \sigma(T) \cap i \mathbf{R}=\varnothing\right\}$ into the set of discrete integers.

PrOOF. This follows immediately from the continuity of $\sigma(T)$. A standard argument may run as follows: Suppose $T \in \mathcal{M}_{n}$ with $\sigma(T) \cap i \mathbf{R}=\varnothing$. Let $f_{T}(\lambda)$ be the characteristic polynomial $\operatorname{det}(T-\lambda I)$. Let $\Gamma$ be any simple closed curve in the open right half-plane such that $\sigma_{+}(T)$ lies completely inside $\Gamma$. Then

$$
\begin{aligned}
\operatorname{sgnt} T & =\#\left\{\text { zeros of } f_{T}(\lambda) \text { inside } \Gamma\right\}-\#\left\{\text { zeros of } f_{T}(\lambda) \text { outside } \Gamma\right\} \\
& =2 \times \#\left\{\text { zeros of } f_{T}(\lambda) \text { inside } \Gamma\right\}-n \\
& =\frac{1}{\pi i} \int_{\Gamma} \frac{f_{T}^{\prime}(\lambda)}{f_{T}(\lambda)} d \lambda-n
\end{aligned}
$$

and the continuity follows.

LEMMA 2. Suppose $X$ and $J$ are square matrices of the same order. If $J$ is a selfadjoint unitary matrix (i.e., $J=J^{*}=J^{-1}$ ) and $\|J-X\|<1$, then $J$ and $X$ have the same signature.

ProOF. First note that whenever $T$ is a matrix with $\|J-T\|<1$, then $\sigma(T) \cap$ $i \mathbf{R}=\varnothing$. This follows from the fact if $T \nu=i a \nu$ with $a \in \mathbf{R}$ and $\|\nu\|=1$, then

$$
\begin{aligned}
\|J-T\|^{2} & \geq\|(J-T) \nu\|^{2}=\|(J-i a I) \nu\|^{2}=\left\langle(J-i a I)^{*}(J-i a I) \nu, \nu\right\rangle \\
& =\left\langle\left(1+a^{2}\right) I \nu, \nu\right\rangle=1+a^{2} \geq 1
\end{aligned}
$$

would lead to a contradiction. 
Now let $T_{t}=t X+(1-t) J$ with $t \in[0,1]$. Then $\left\|J-T_{t}\right\|=t\|J-X\|<t \leq 1$. From above, $\sigma\left(T_{t}\right) \cap i \mathbf{R}=\varnothing$ for each $t$. By Lemma $1, t \rightarrow \operatorname{sgnt} T_{t}$ is a continuous function with integer values. Thus $J=T_{0}$ and $X=T_{1}$ have the same signature.

The following lemma is likely known to some readers. Since there is no handy reference, a proof is provided.

LEMMA 3. Suppose $A, B, C, D$ are $n \times n$ matrices and $A$ is invertible. Then $\operatorname{det}\left[\begin{array}{ll}A & B \\ C & D\end{array}\right]=\operatorname{det}\left(D A-C A^{-1} B A\right)$.

ProOF. By direct computation,

$$
\left[\begin{array}{ll}
A & B \\
C & D
\end{array}\right]=\left[\begin{array}{cc}
I & 0 \\
C A^{-1} & I
\end{array}\right]\left[\begin{array}{cc}
I & 0 \\
0 & D-C A^{-1} B
\end{array}\right]\left[\begin{array}{cc}
I & B \\
0 & I
\end{array}\right]\left[\begin{array}{cc}
A & 0 \\
0 & I
\end{array}\right] .
$$

Thus

$\operatorname{det}\left[\begin{array}{ll}A & B \\ C & D\end{array}\right]=\operatorname{det} I \cdot \operatorname{det}\left(D-C A^{-1} B\right) \cdot \operatorname{det} I \cdot \operatorname{det} A=\operatorname{det}\left(D A-C A^{-1} B A\right)$.

LEMMA 4. If $R, S, T$ are $n \times n$ matrices with $R S=S R$, then the signature of $\left[\begin{array}{cc}R & S \\ T & -R\end{array}\right]$ is zero.

ProOF. Let $X=\left[\begin{array}{cc}R & S \\ T & -R\end{array}\right]$. For each $\lambda \notin \sigma(R)$, we have

$$
\operatorname{det}(X-\lambda I)=\operatorname{det}\left[\begin{array}{cc}
R-\lambda I & S \\
T & -R-\lambda I
\end{array}\right]=\operatorname{det}\left(\lambda^{2} I-R^{2}-T S\right)
$$

by Lemma 3 and the fact $R S=S R$. Similarly,

$$
\operatorname{det}(-X-\lambda I)=\operatorname{det}\left[\begin{array}{cc}
-R-\lambda I & -S \\
-T & R-\lambda I
\end{array}\right]=\operatorname{det}\left(\lambda^{2} I-R^{2}-T S\right) .
$$

Thus $X$ and $-X$ have the same characteristic polynomial. Therefore sgnt $X=$ $\operatorname{sgnt}(-X)=0$.

3. Example and remarks. In order to avoid computational complexity, we present a $2 n \times 2 n$ matrix with nice entries.

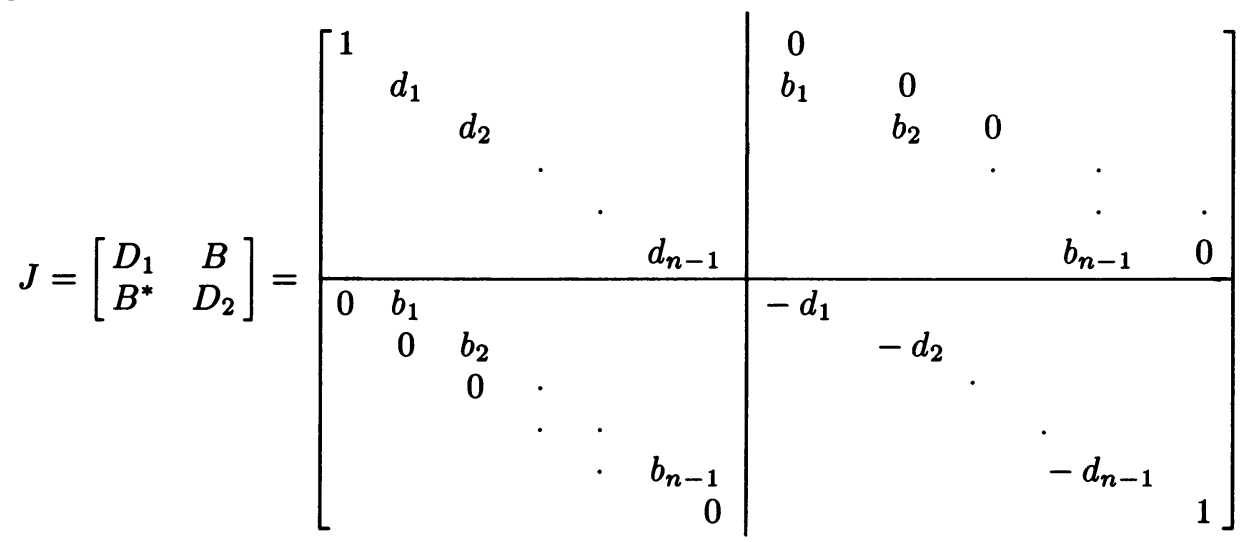

where $d_{j}^{2}+b_{j}^{2}=1, d_{j}=(n-2 j) / n, b_{j}=2[j(n-j)]^{1 / 2} / n$. It is readily seen that $J^{2}=\left[\begin{array}{ll}I & 0 \\ 0 & I\end{array}\right]$, and $D_{1}+D_{2}=2 I / n$. 
THEOREM (EXAMPLE). For each integer $n>1$, there exist $A, B \in \mathcal{M}_{n}$ with $\|A\|=1-1 / n,\|B\| \leq 1,\|A B-B A\| \leq 2 / n$, but $\|A-R\|+\|B-S\| \geq 1-1 / n$ for all commutingf $R, S \in \mathcal{M}_{n}$.

ProOF. Let $B, D_{1}$, and $D_{2}$ be the $n \times n$ matrices displayed above and let $A=D_{1}-I / n=-D_{2}+I / n$. Then it is evident that $\|A\|=1-1 / n,\|B\| \leq 1$, and

$$
J=\frac{1}{n}\left[\begin{array}{ll}
I & 0 \\
0 & I
\end{array}\right]+\left[\begin{array}{cc}
A & B \\
B^{*} & -A
\end{array}\right]
$$

Since $J^{2}=\left[\begin{array}{ll}I & 0 \\ 0 & I\end{array}\right]$, it follows that $(I / n+A) B+B(I / n-A)=0$, thus $\|A B-B A\|=$ $\|2 B / n\| \leq 2 / n$. Furthermore, $J$ is a selfadjoint unitary matrix with sgnt $J=$ trace $J=2$.

Now suppose $R, S \in \mathcal{M}_{n}$ with $R S=S R$. Then by Lemma $4, X=\left[\begin{array}{cc}R & S \\ S^{*} & -R\end{array}\right]$ has zero signature. By Lemma 2, we deduce that

$$
1 \leq\|J-X\|=\left\|\frac{1}{n}\left[\begin{array}{ll}
I & 0 \\
0 & I
\end{array}\right]+\left[\begin{array}{cc}
A-R & B-S \\
B^{*}-S^{*} & -A+R
\end{array}\right]\right\| \leq \frac{1}{n}+\|A-R\|+\|B-S\|,
$$

and thus $\|A-R\|+\|B-S\| \geq 1-1 / n$ as desired.

REMARK 1. The inequality $\|A-R\|+\|B-S\| \geq 1-1 / n$ is a sharp estimate since $(R, S)=(0, B)$ yields $\|A-R\|+\|B-S\|=1-1 / n$. This also means that $(0, B)$ is a commuting approximant to $(A, B)$.

REMARK 2. We can associate each pair of nontrivial matrices $(A, B)$ with two real numbers in $[0,1]$

and

$$
\delta(A, B)=\frac{\|A B-B A\|}{2\|A\| \cdot\|B\|}
$$

$$
\varepsilon(A, B)=\frac{\inf \{\|A-R\|+\|B-S\|: \text { all commuting pairs }(R, S)\}}{\min \{\|A\|,\|B\|\}} .
$$

The fact $\varepsilon(A, B) \leq 1$ follows from the computation with $(R, S)=(A, 0)$ or $(0, B)$. Roughly speaking, $\delta(A, B)$ indicates the amount of noncommutativity while $\varepsilon(A, B)$ indicates how far from commuting. Thus, for the specifically constructed example $(A, B)$ of the Theorem, we have $\delta(A, B) \leq 1 /(n-1)$, and $\varepsilon(A, B)=1$-this means that $(A, B)$ is "almost commuting," yet $(A, B)$ is "extremely far" (or "farthest") from commuting pairs.

REMARK 3 . The study of perturbations of operator algebras is much related to the study of approximations of operators. Thus, the following problem arises naturally: Must almost commutative matrix algebras be near to commutative matrix algebras? More precisely, we ask: For each $\varepsilon>0$, does there exist $\delta=\delta(\varepsilon)>0$, such that for each integer $n$, if $A$ is a subalgebra of $\mathcal{M}_{n}$ with $\left\|A_{1} A_{2}-A_{2} A_{1}\right\| \leq \delta\left\|A_{1}\right\|\left\|A_{2}\right\|$ for all $A_{1}, A_{2} \in A$, then there is a commutative algebra $B \subseteq \mathcal{M}_{n}$ with $\operatorname{dist}(A, B) \leq \varepsilon\|A\|$ for each $A \in A$ ?

\section{REFERENCES}

1. J. J. Bastian and K. J. Harrison, Subnormal weighted shifts and asymptotic properties of normal operators, Proc. Amer. Math. Soc. 42 (1974), 475-479.

2. I. D. Berg, On operators which almost commute with the shift, J. Operator Theory 11 (1984), 365-377. 
3. I. D. Berg and K. R. Davidson, A quantitative version of the Brown-Douglas-Fillmore theorem, preprint.

4. I. D. Berg and C. L. Olsen, A note on almost commuting operators, Proc. Roy. Irish Acad. Sect. A 81 (1981), 43-47.

5. M.-D. Choi, Lifting projections from quotient $C^{*}$-algebras, J. Operator Theory 10 (1983), 2130.

6. M.-D. Choi and E. Christensen, Completely order isomorphic and close $C^{*}$-algebras need not be *-isomorphic, Bull. London Math. Soc. 15 (1983), 604-610.

7. K. R. Davidson, Almost commuting Hermitian matrices, Math. Scand. 56 (1985), 222-240.

8. P. R. Halmos, Some unsolved problems of unknown depth about operators on Hilbert space, Proc. Roy. Soc. Edinburgh Sect. A 76 (1976), 67-76.

9. T. Loring. The torus and non-commutative topology, Thesis, University of California, Berkeley, 1986.

10. W. A. J. Luxemburg and R. F. Taylor, Almost commuting matrices are near commuting matrices, Indag. Math. 32 (1970), 96-98.

11. C. Pearcy and A. Shields, Almost commuting matrices, J. Funct. Anal. 33 (1979), 332-338.

12. P. Rosenthal, Are almost commuting matrices near commuting matrices? Amer. Math. Monthly 76 (1969), 925-926.

13. D. Vioculescu, Asymptotically commuting finite rank unitaries without commuting approximants, Acta Sci. Math. (Szeged) 45 (1983), 429-431.

Department of MAThematics, University of TORONTO, TORONTO, CANAda M5S $1 \mathrm{~A} 1$ 